\title{
Monte Carlo Simulation on Triple Layer Thin Film Spin Lattice in Extended Heisenberg Model
}

\author{
E. BÎRSAN* \\ Physics Department, "Lucian Blaga" University \\ Dr I. Ratiu Str., No. 5-7, 550024, Sibiu, Romania \\ (Received August 6, 2008; revised version October 6, 2008)
}

\begin{abstract}
In this paper the magnetic behavior of triple layer thin film was investigated by Monte Carlo simulations. We fixed an antiferromagnetic arrangement of the lattice spins for the basal layer of the film and we studied the basal layer magnetic ordering influence on the two other layers, by calculating the out-of-plane and the in-plane magnetization, the out-of-plane and the in-plane magnetic susceptibility and the specific heat. We found out five magnetic ordering phases of the sample: ferromagnetic, antiferromagnetic, mixed phase ordering, paramagnetic and $X Y$-like magnetic ordering of the spins, respectively.
\end{abstract}

PACS numbers: 75.10.Hk, 75.70.Ak, 75.40.Mg

\section{Introduction}

For the last years there has being an increasing interest in ultrathin magnetic films area [1-3] due to their important technical applications [4]. The magnetization processes in ultrathin films, used in data storage devices technology, are dominated by the balance between the surface and the shape anisotropies: the surface anisotropy arises from the symmetry breaking in the direction perpendicular to the film plane $[5,6]$ and favors a magnetization along this direction, whereas the shape anisotropy, which results from the long-range dipoledipole interactions, favors the in-plane magnetization. A lot of magnetic domains characterized by the competition between dipolar long-range interactions and strong anisotropies perpendicular to the plane of the film were observed experimentally $[7,8]$. At the same time, many theoretical works have been done on the morphology and stability of these magnetic structures $[9,10]$. We also remark the great interest of the researchers about the ferromagnetic/antiferromagnetic ( $\mathrm{FM} / \mathrm{AFM})$ thin films reason to numerous special physical phenomena which appear in these systems. For example, magnetic properties of FM/AFM bilayer can be drastically different from those of free FM and AFM films, one of the established effects being a unidirectional shift (exchange bias) and a significant increase in the coercivity. The blocking temperature $\left(T_{\mathrm{B}}\right)$ below which these effects are observed, is comparable to the bulk Néel temperature when the AFM film is thick, but can be considerably lower when the AFM film is thin $[11,12]$. The relative recent neutron ex-

\footnotetext{
* e-mail: beneugen10@yahoo.com
}

periments [13] on $\mathrm{CoO} / \mathrm{Fe}_{3} \mathrm{O}_{4}$ have found that the $\mathrm{AFM}$ shows signs of ordering above $T_{\mathrm{B}}$. This indicates that the proximity of the FM influences the phase transition in the AFM in a way that cannot be predicted from studying free AFM films. The interfacial magnetic phenomena also rouse a great interest in thin film domain due to the most interesting physical properties that can arise from parameter's discontinuity. In this paper we investigate the triple-layer thin film magnetic behavior, in extended anisotropic Heisenberg model, using Monte Carlo simulations for different parameters settings. In this order we try to find out the magnetic properties of this square spins lattice disposed on three layers, the first of them being characterized by antiferromagnetic spin ordering. We want to detect the first layer's antiferromagnetic arrangement of the spins influence on the other layers respecting the magnetic state ordering, for different physical parameter's settings and we principally focus our attention on the investigation of different potential magnetic ordering phases of the lattice spins occurrence.

The paper is organized as follows: Sect. 2 is devoted to the description of the physical model we use, in Sect. 3 we describe the numerical Monte Carlo algorithm and present the details of this and the physical quantities necessary to elaborate our simulations, in Sects. 4 we display the numerical results for different parameters settings, concluding remarks being given in Sect. 5 .

\section{Physical model}

We consider in this paper a triple-layer thin film characterized by the following extended anisotropic Heisenberg Hamiltonian: 


$$
\begin{aligned}
H & =-\sum_{\langle i, j\rangle} J_{\alpha_{i} \beta_{j}} \boldsymbol{S}_{i} \cdot \boldsymbol{S}_{j}-\sum_{\langle i, j\rangle} A_{\alpha_{i} \beta_{j}} S_{i z} S_{j z} \\
& +\sum_{i, j} D_{\alpha_{i} \beta_{j}} T_{i j} .
\end{aligned}
$$

The first term denotes the direct exchange interaction between the nearest neighbor spins $(\langle i, j\rangle$ meaning a summation over the nearest neighbor), the second term is related to the exchange anisotropy interaction between the nearest-neighbor spins and behaves as easy-axis anisotropy since it tends to align the spins in the $O Z$ direction in order to lower the energy of the system and the third term in Hamiltonian (1) corresponds to the long-range dipolar interaction between the spins. In the last term the dipolar interaction (characterized by the parameter $\left.D_{\alpha \beta}\right)$ is given by the expression

$$
T_{i j}=\frac{\boldsymbol{S}_{i} \cdot \boldsymbol{S}_{j}}{r_{i j}^{3}}-3 \frac{\left(\boldsymbol{S}_{i} \cdot \boldsymbol{r}_{i j}\right)\left(\boldsymbol{S}_{j} \cdot \boldsymbol{r}_{i j}\right)}{r_{i j}^{5}},
$$

where $r_{i j}$ defines the relative distance between the spins. In the next, we index the interaction parameters dependent on the layer the spins belong to. Thus, we index the antiferromagnetic ordered layer by " 0 " and the other two by "1" and "2", respectively. $J_{\alpha \beta}$ represents the direct exchange interaction constant, $A_{\alpha \beta}$ is the easy-axis anisotropy interaction parameter, and $D_{\alpha \beta}$ is the dipolar interaction between spins parameter (long-range interaction). All parameters in the next are being indexed according to the layer which the specified spins are part of $\left(\alpha_{i}, \beta_{j}=0,1,2\right.$ in accordance with the layer's index $)$. We also consider the distance between the intra-layer spins and the inter-layers distance are the same $(a)$, the spins sizes being $S_{0}=S_{1}=S_{2}=S=1$ (arranged in a simple cubic lattice with a (001) face). In the next, we index all the model parameters in accordance with the positions of the spins in the three layers. We set for direct exchange interaction for the base layer (" 0 ") $J_{00}=-J=-1$, in the next paragraphs $J S^{2}$ and $J S^{2} / k_{\mathrm{B}}$ being the units for energy and temperature, respectively.

\section{Numerical algorithm}

In order to obtain the properties of the triple-layer magnetic films in the physical model presented above, we have used the Monte Carlo simulation [14, 15], by applying the standard Metropolis algorithm [16] on our defined square spins lattice. Typically, $10^{3}-10^{4} \mathrm{MC}$ steps per spin are used for equilibration and $10^{3}$ steps for spin are used for calculating the interesting physical average quantities. We also have used open boundary conditions (OBC) and we set $k_{\mathrm{B}}=1$ for the Boltzmann constant. In order to investigate the physical properties of the system, we need the statistical average of the energy per spin

$$
\langle E\rangle=\frac{\langle H\rangle}{N},
$$

where \langle\rangle denotes the statistical average, which is calculated by taking in account the partition function over the Monte Carlo steps energies and $N=N_{x} N_{y} N_{z}$ represents the number of sites of the lattice. For the specific heat calculation we use the expression given by the energy dispersion

$$
c=N \frac{\left\langle E^{2}\right\rangle-\langle E\rangle^{2}}{k_{\mathrm{B}} T^{2}} .
$$

We also use for our simulations the out-of-plane $(O Z)$ and in-plane $(X O Y)$ magnetic susceptibility, given by the expressions

$$
\begin{aligned}
& \chi_{z}=N \frac{\left\langle m_{z}^{2}\right\rangle-\left\langle m_{z}\right\rangle^{2}}{k_{\mathrm{B}} T}, \\
& \chi_{x y}=N \frac{\left\langle m_{x y}^{2}\right\rangle-\left\langle m_{x y}\right\rangle^{2}}{k_{\mathrm{B}} T},
\end{aligned}
$$

where $m_{z}, m_{x y}$ are the $O Z$ and $X O Y$ components of the magnetization vector

$$
\boldsymbol{m}=\frac{\sum_{i=1}^{N} \boldsymbol{S}_{i}}{N},
$$

$\boldsymbol{S}_{i}$ meaning the spin at the site " $i$ ". The components of the magnetization are given by the following expressions:

$$
\begin{aligned}
& m_{k}=\frac{\sum_{i=1}^{N} S_{i k}}{N}, \quad k=x, y, z, \\
& m_{x y}=\sqrt{m_{x}^{2}+m_{y}^{2}} .
\end{aligned}
$$

In order to investigate the potential antiferromagnetic ordering we also define the $O Z$ component of the staggered magnetization, by the expression

$$
m_{s z}=\frac{\sum_{n}(-1)^{i+j} S_{z n}}{N} .
$$

\section{Numerical results}

As we already assigned in the previous sections, the basal layer spins are characterized by a fixed antiferromagnetic ordering and the two other layers are characterized by the Hamiltonian mentioned in our model presentation. The three interactions are extended also on the basal layer, but their magnetic spin ordering is assumed fixed (antiferromagnetic). Only the two superior layers (" 1 " and " 2 ") contributions are taken into account to physical quantities we calculate in our numerical simulations (the sample to investigate).

\subsection{Ferromagnetism-paramagnetism transition}

In this paragraph we set for the lattice size $N_{x}=N_{y}=$ 50 , distance between the adjacent spins $a=1$ (this being in the same time the inter-layer distances) and the other parameter values are given by: $J_{11}=J_{22}=J=1$, $J_{01}=J_{12}=J^{\prime}=0.75, A_{11}=A_{22}=A=1$ and $A_{01}=A_{12}=A^{\prime}=1.1,1.2$, and 1.3 , respectively, $D_{11}=D_{22}=D=0.1, D_{01}=D_{12}=D^{\prime}=0.1$. In Fig. 1 (top) we plot the out-of-plane magnetization (in the next we represent the magnetizations normalized to unity and consequently dimensionless) versus temperature for threeversus temperature for three different 


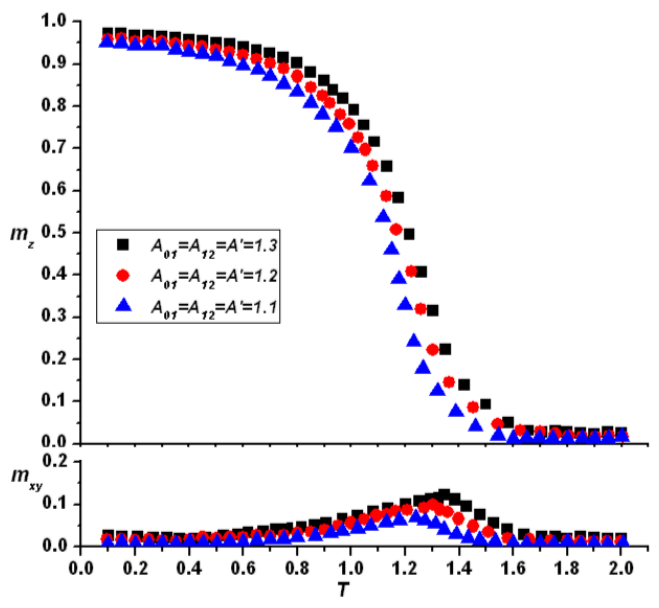

Fig. 1. Out-of-plane (top) and in-plane (bottom) magnetization of the sample versus temperature for $J_{11}=$ $J_{22}=J=1, J_{01}=J_{12}=J^{\prime}=0.75, A_{11}=A_{22}=$ $A=1, D_{11}=D_{22}=D=0.1, D_{01}=D_{12}=D^{\prime}=0.1$, for different $A_{01}=A_{12}=A^{\prime}$ values.

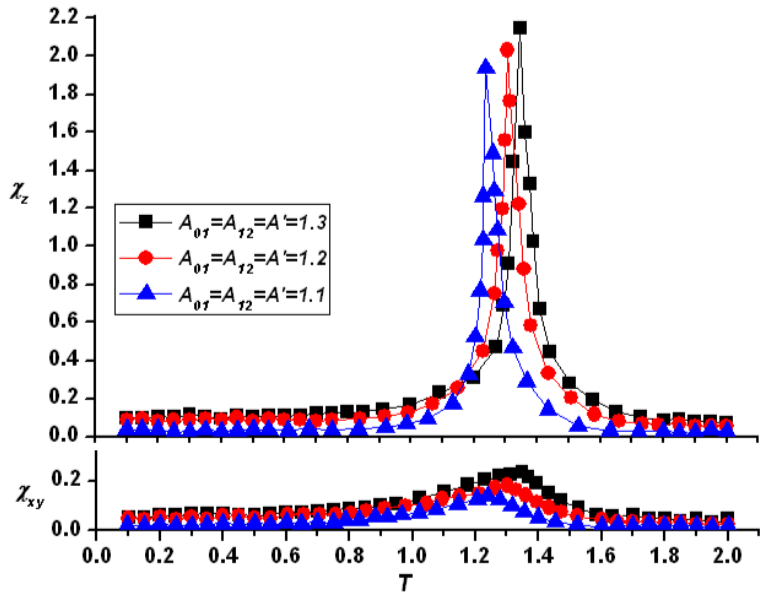

Fig. 2. Out-of-plane (top) and in-plane (bottom) magnetic susceptibility of the sample versus temperature for $J_{11}=J_{22}=J=1, J_{01}=J_{12}=J^{\prime}=0.75$, $A_{11}=A_{22}=A=1, D_{11}=D_{22}=D=0.1$, $D_{01}=D_{12}=D^{\prime}=0.1$, for different $A_{01}=A_{12}=A^{\prime}$ values.

inter-layer anisotropy parameters which suggests a ferromagnetic ordering of the sample at low temperature, at large temperature this magnetic ordering of the spins disappears. The phase ferromagnetism-paramagnetism transition (FM-PM) is characterized by the critical temperature which slightly shifts to lower values along with decreasing anisotropy parameter, the critical temperature being well approximated by the inflection point (the second-order derivative changes the sign marked with the arrows) of the magnetization graph.

In Fig. 1 (bottom) we show the out-of-plane magnetization versus temperature and we can observe that the $X Y$ magnetization of the spins is practically absent that con-

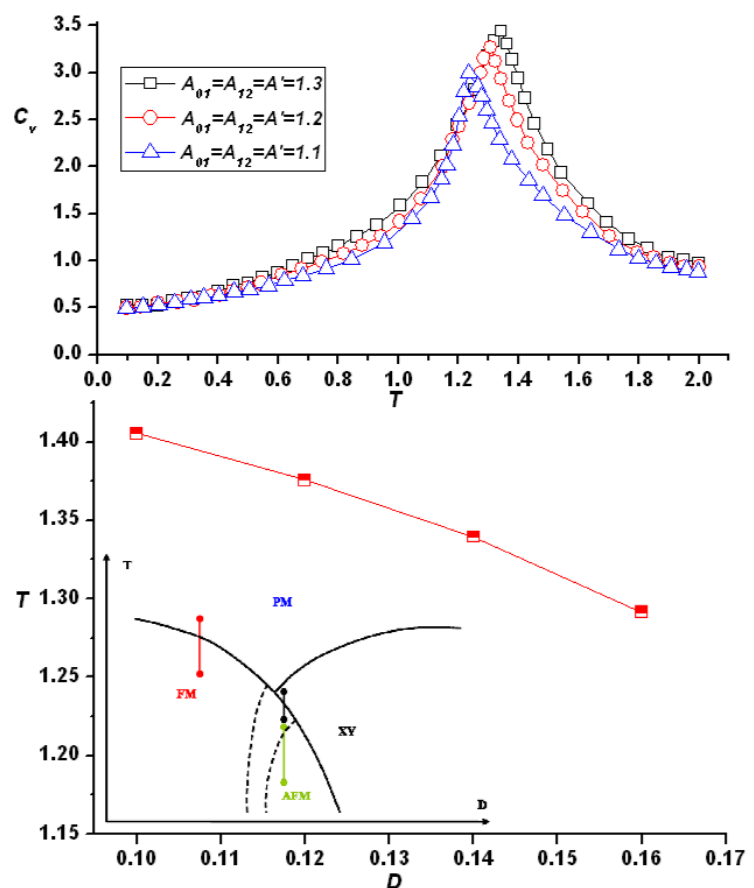

Fig. 3. Specific heat of the sample versus temperature (top) for $J_{11}=J_{22}=J=1, J_{01}=J_{12}=J^{\prime}=0.75$, $A_{11}=A_{22}=A=1, D_{11}=D_{22}=D=0.1$, $D_{01}=D_{12}=D^{\prime}=0.1$, for different $A_{01}=A_{12}=A^{\prime}$ values and $\mathrm{FM} \rightarrow \mathrm{PM}$ phase diagram line, along with the dipolar interaction parameter (bottom); the inset presents the qualitative phase diagram we expect for the sample.

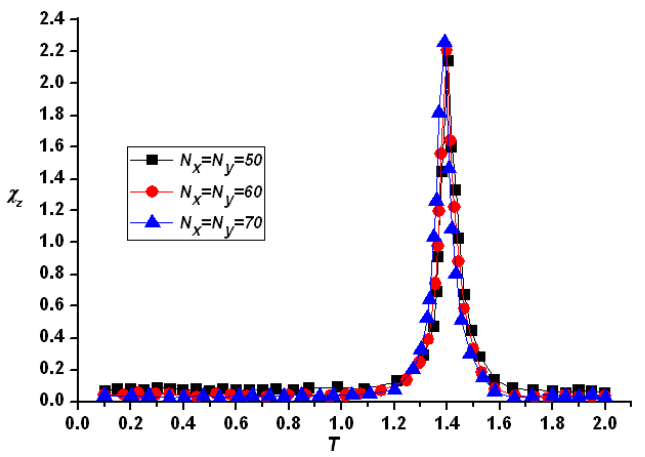

Fig. 4. Out-of-plane magnetic susceptibility of the sample versus temperature for $J_{11}=J_{22}=J=1$, $J_{01}=J_{12}=J^{\prime}=0.75, A_{11}=A_{22}=A=1$, $A_{01}=A_{12}=A^{\prime}=1.3, D_{11}=D_{22}=D=0.1$, $D_{01}=D_{12}=D^{\prime}=0.1$, for different lattice size values.

firms the out-of-plane magnetization of these. In Fig. 2, we present the out-of-plane (top) and in-plane (bottom) magnetic susceptibility, where we detect the same decreasing critical temperature (along with the amplitude subtraction of the magnetic susceptibility peaks amplitude) as the interlayer easy-axis anisotropy parameter decreases, this behavior confirming that the anisotropy 
interaction enlargement favors the ferromagnetic spin ordering in the sample, for parameter setting presented above, although the basal layer is characterized by an antiferromagnetic arrangement of the spins, the preferential orientations of the FM spins observed here resulting from the exchange coupling to the AFM film [17].

Figure 3 (top) depicts the specific heat of the system versus temperature for the three different anisotropy parameter values. In Fig. 3 (bottom) we present four points of the phase separation line $(\mathrm{FM} \rightarrow \mathrm{PM})$, critical point being obtained for different dipolar interaction straight Monte Carlo simulations for interlayer anisotropy parameter $A^{\prime}=1.3$, the other parameters being those specified in this section; in the inset we plot the qualitative phase diagram we expect for our thin film, where we display the magnetic phase of the physical system: the FM phase, the disordered phase (PM), the mixed phase (MP) situated between the broken lines, the AFM ordering and the in-plane spin arrangement $(X Y)$.

In Fig. 4 we present the finite size effect, regarding the $\mathrm{FM} \rightarrow \mathrm{PM}$ phase transition (transition symbolized by the FM segment in the phase diagram - Fig. 3, bottom), by taking into account the out-of-plane magnetic susceptibility behavior.

\subsection{Antiferromagnetism - mixed phase transition}

In this paragraph we maintain for the lattice size $N_{x}=N_{y}=50$ and for distance between the adjacent spins $a=1$ (this being in the same time the inter-layer distances) and for the other parameter values we choose:

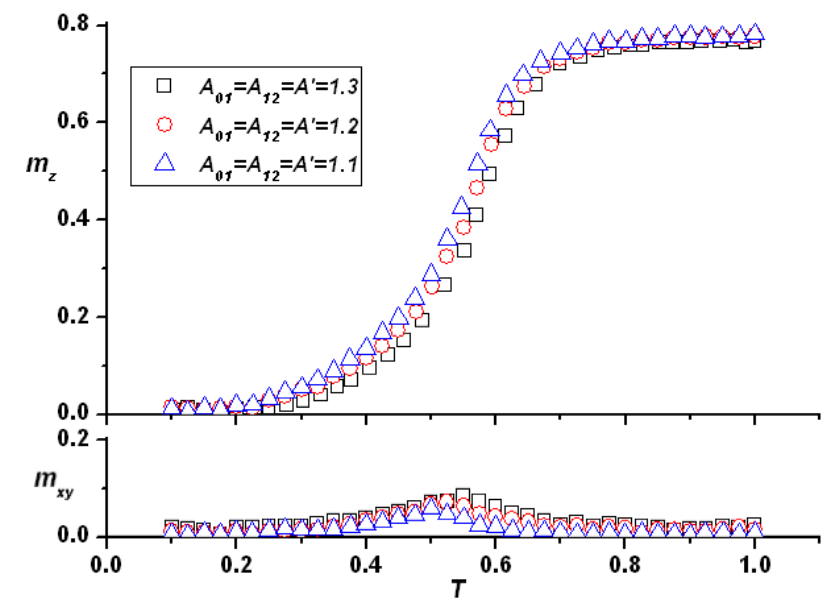

Fig. 5. Out-of-plane (top) and in-plane (bottom) magnetization of the sample versus temperature for $J_{11}=$ $J_{22}=J=1, J_{01}=J_{12}=J^{\prime}=0.75, A_{11}=A_{22}=$ $A=1, D_{11}=D_{22}=D=0.175, D_{01}=D_{12}=D^{\prime}=0.2$, for different $A_{01}=A_{12}=A^{\prime}$ values.

$J_{11}=J_{22}=J=1, J_{01}=J_{12}=J^{\prime}=0.75, A_{11}=A_{22}=$ $A=1$ and $A_{01}=A_{12}=A^{\prime}=1.1,1.2$, and 1.3, respectively, $D_{11}=D_{22}=D=0.175, D_{01}=D_{12}=D^{\prime}=0.2$. In Fig. 5 we present the out-of-plane (top) and in-plane (bottom) magnetization of the sample for the above presented model parameters setting.

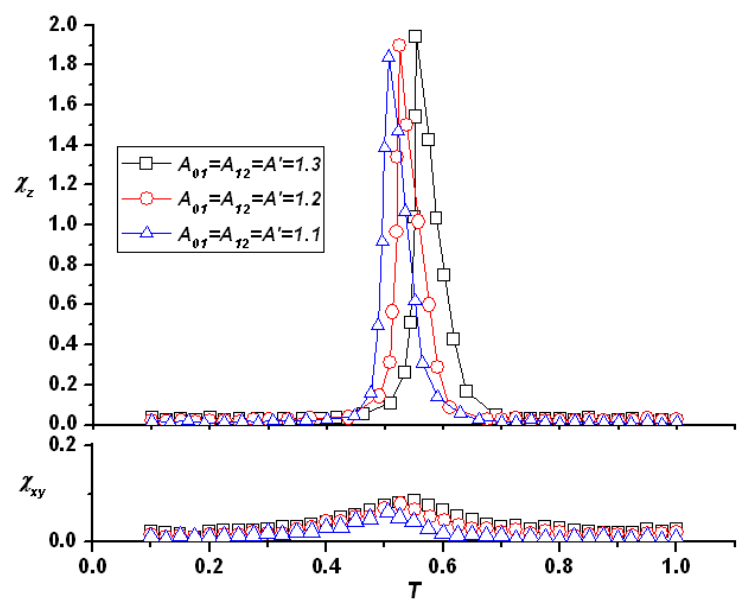

Fig. 6. Out-of-plane (top) and in-plane (bottom) magnetic susceptibility of the sample versus temperature for $J_{11}=J_{22}=J=1, J_{01}=J_{12}=J^{\prime}=0.75$ $A_{11}=A_{22}=A=1, D_{11}=D_{22}=D=0.175$ $D_{01}=D_{12}=D^{\prime}=0.2$, for different $A_{01}=A_{12}=A^{\prime}$ values.

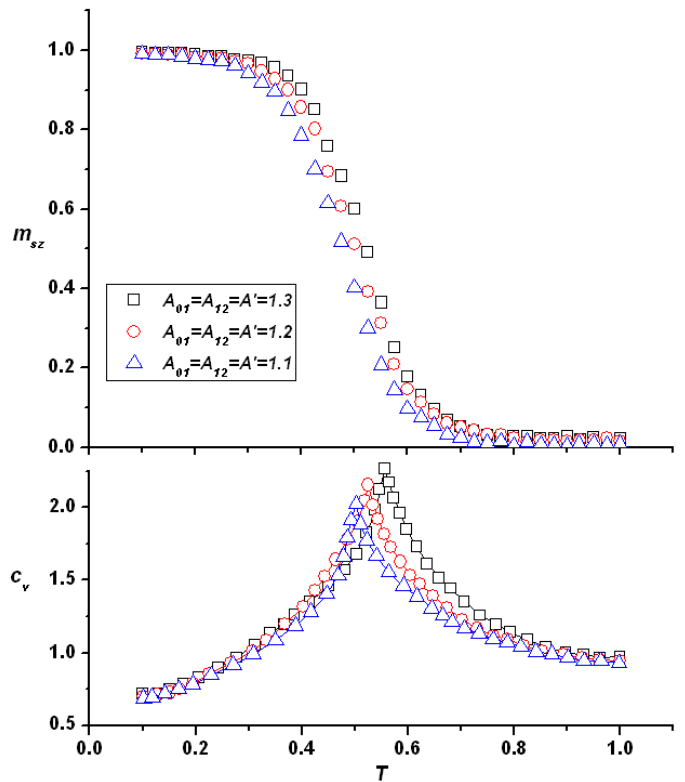

Fig. 7. Out-of-plane staggered magnetization (top) and specific heat (bottom) of the sample versus temperature for $J_{11}=J_{22}=J=1, J_{01}=J_{12}=J^{\prime}=0.75$, $A_{11}=A_{22}=A=1, D_{11}=D_{22}=D=0.175$, $D_{01}=D_{12}=D^{\prime}=0.2$, for different $A_{01}=A_{12}=A^{\prime}$ values.

The out-of-plane magnetization vanishes at low temperature and increases abruptly in the range of temperature $T=[0.5,0.6]$, whereas the in-plane magnetization is quasi-absent. To elucidate the magnetic phase of the sample we show in Fig. 6 the out-of-plane (top) and in-plane (bottom) magnetic susceptibility of the system, where we observe the one peak behavior of the out-of- 
-plane magnetic susceptibility that suggests a phase transition in the same range of temperature (the AFM segment in the phase diagram presented in Fig. 3, bottom). The peak's amplitude slightly decreases together with the critical temperature when the easy-axis anisotropy $A^{\prime}$ parameter value decreases.

The specific heat bearing (Fig. 7, right) confirms the assumed phase transition but to trash out the nature of this, we calculate the staggered out-of-plane magnetization (Fig. 7, left) and we find out that the system suffers an AFM $\rightarrow$ MP transition (taking into account the out-of-plane uniform and the staggered magnetization graphs), the MP consisting in ferromagnetic domains enclosed by disorder phase regions, as we have remarked in our previous paper [18], where we have found out the induced antiferromagnetic phase and the mixed phase appearance in a double layer spin lattice, which is a more simple model we have used to investigate this kind of $\mathrm{AFM} / \mathrm{FM}$ interfaces. In this more elaborated model of the triple layer lattice, the influence of the antiferromagnetic basal layer on the superior layers is evidently different, taking into account the asymmetry of the system.

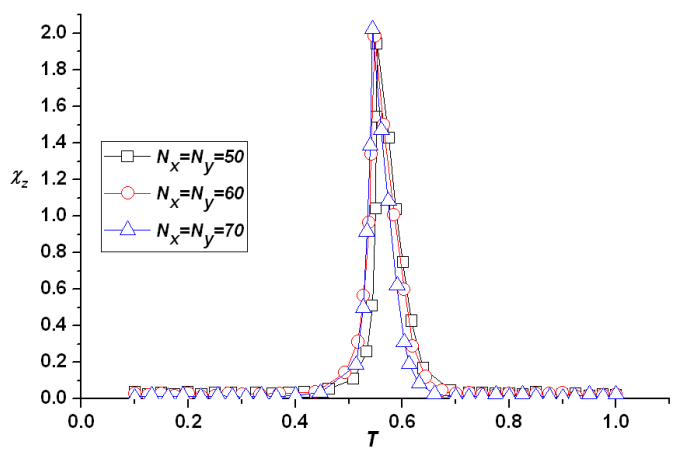

Fig. 8. Out-of-plane magnetic susceptibility of the sample versus temperature for $J_{11}=J_{22}=J=1$, $J_{01}=J_{12}=J^{\prime}=0.75, A_{11}=A_{22}=A=1$, $A_{01}=A_{12}=A^{\prime}=1.3, D_{11}=D_{22}=D=0.175$, $D_{01}=D_{12}=D^{\prime}=0.2$, for different lattice size values.

Now we focus on the finite size effect on the out-of-plane magnetic susceptibility (Fig. 8) for three lattice sizes $\left(N_{x}=N_{y}=50,60,70\right.$, respectively) and we observe detect a very slight decrement of the critical temperature along with the lattice size increasing. The calculus algorithm is described above (Eq. (11)) and similar in both of the cases (for temperature and out-of-plane magnetic susceptibility amplitude).

\subsection{Mixed phase $-X Y$ ordering transition}

In this section we conserve the same parameters setting as in previous section (4.2), but we choose another temperature range for our numerical simulation: $T=[1.05,1.25]$. Figure 9 shows the out-of-plane (top) and in-plane (bottom) magnetization of the sample where one observes a very low out-of-plane component but an abrupt increasing for the in-plane magnetization in high

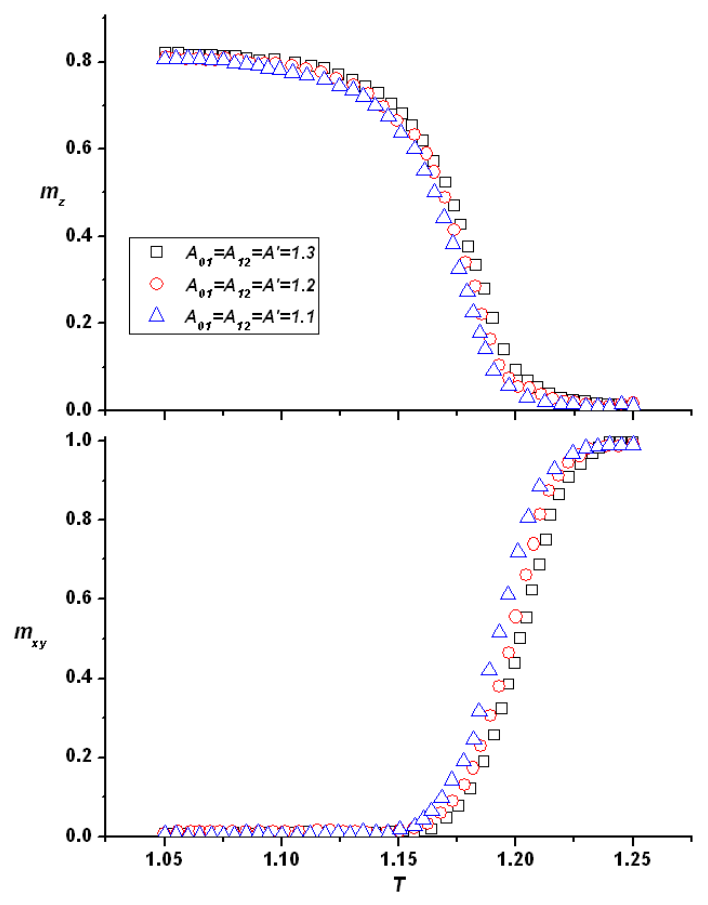

Fig. 9. Out-of-plane (top) and in-plane (bottom) magnetization of the sample versus temperature for $J_{11}=$ $J_{22}=J=1, J_{01}=J_{12}=J^{\prime}=0.75, A_{11}=A_{22}=$ $A=1, D_{11}=D_{22}=D=0.175, D_{01}=D_{12}=D^{\prime}=0.2$, for different $A_{01}=A_{12}=A^{\prime}$ values.

temperature region that suggests a reorientation of the spin ( $X Y$ ordering).

In Fig. 10 there are shown the out-of-plane (top) and the in-plane magnetic susceptibility (bottom) of the sample for the physical conditions presented above, in this section and provide the arguments of magnetic ordering phase transition (symbolized by the acute peaks of the graphs), together with the specific heat bearing illustrated in Fig. 11 (bottom). The amplitude of the peaks slightly increases (together with the small enlargement of the critical temperature) along with the inter-plane anisotropy $\left(A^{\prime}\right)$ parameter increasing. Consequently, one can observe that the behavior of the physical quantities mentioned above suggests the out-of-plane preponderant orientation of the lattice spins at low temperature and the reorientation of these at high temperature values of the temperature interval considered in this section (in-plane arrangement of the spins).

In order to elucidate the spin ordering in the low temperature region of the temperature range assumed in this section, we plot in Fig. 11 (top) the out-of-plane staggered magnetization and we detect here the quasi-null values of this for all the temperature spectra we have assumed in this section, the physical presented quantities bearing proposing a mixed phase ordering of the lattice spins for $T \approx[1.05,1,18]$ and the in-plane magnetic ordering of the spins for the other temperature range $[1.18,1.25]$. Thus we proved the presence of the 


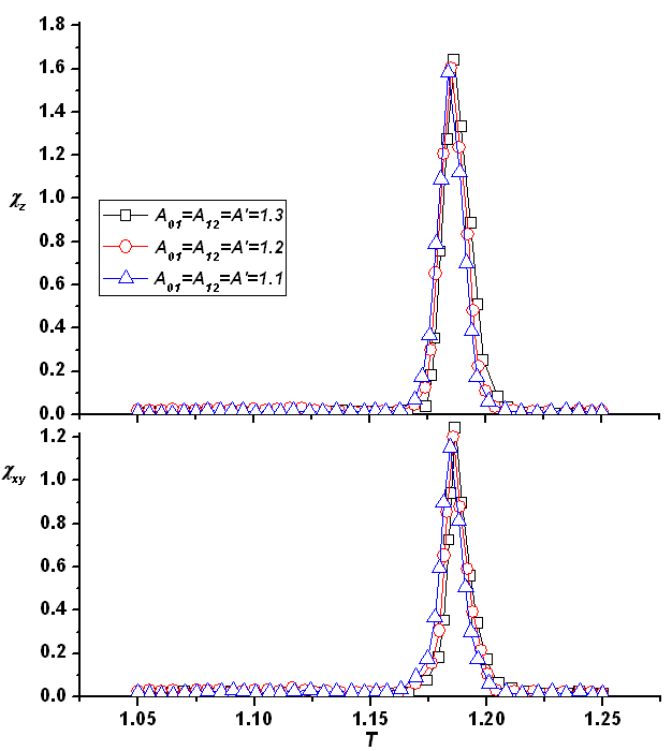

Fig. 10. Out-of-plane (top) and in-plane (bottom) magnetic susceptibility of the sample versus temperature for $J_{11}=J_{22}=J=1, J_{01}=J_{12}=J^{\prime}=0.75$, $A_{11}=A_{22}=A=1, D_{11}=D_{22}=D=0.175$, $D_{01}=D_{12}=D^{\prime}=0.2$, for different $A_{01}=A_{12}=A^{\prime}$ values.

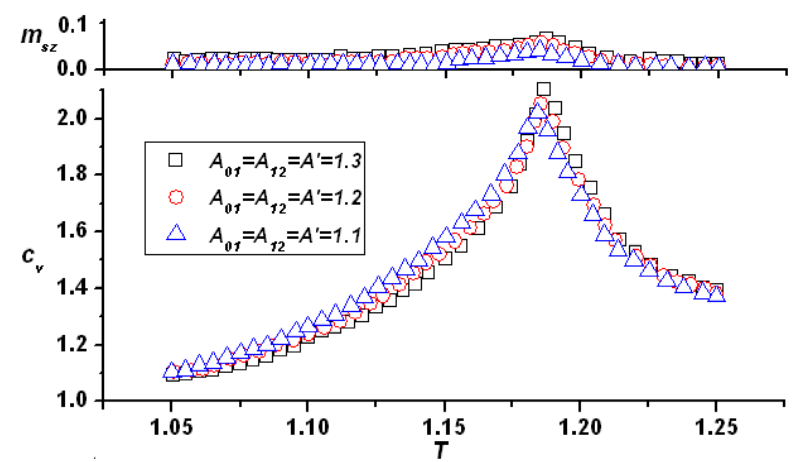

Fig. 11. Out-of-plane staggered magnetization (top) and specific heat (bottom) of the sample versus temperature for $J_{11}=J_{22}=J=1, J_{01}=J_{12}=J^{\prime}=0.75$, $A_{11}=A_{22}=A=1, D_{11}=D_{22}=D=0.175$, $D_{01}=D_{12}=D^{\prime}=0.2$, for different $A_{01}=A_{12}=A^{\prime}$ values.

$\mathrm{MP} \rightarrow \mathrm{XY}$ phase transition (black segment in phase diagram - see inset of Fig. 3, bottom) of our system for the physical conditions indicated above. Certainly, if the temperature thenceforth increases, the physical system will pass into paramagnetic phase, where the spins are randomly arranged.

In this paragraph we investigate the finite size effect on magnetic out-of-plane (Fig. 12, top) and in-plane (Fig. 12, bottom) magnetic susceptibility, for three different lattice sizes.

Thus, we observe a very small relative variation of the critical temperature (that presents a slight decrease

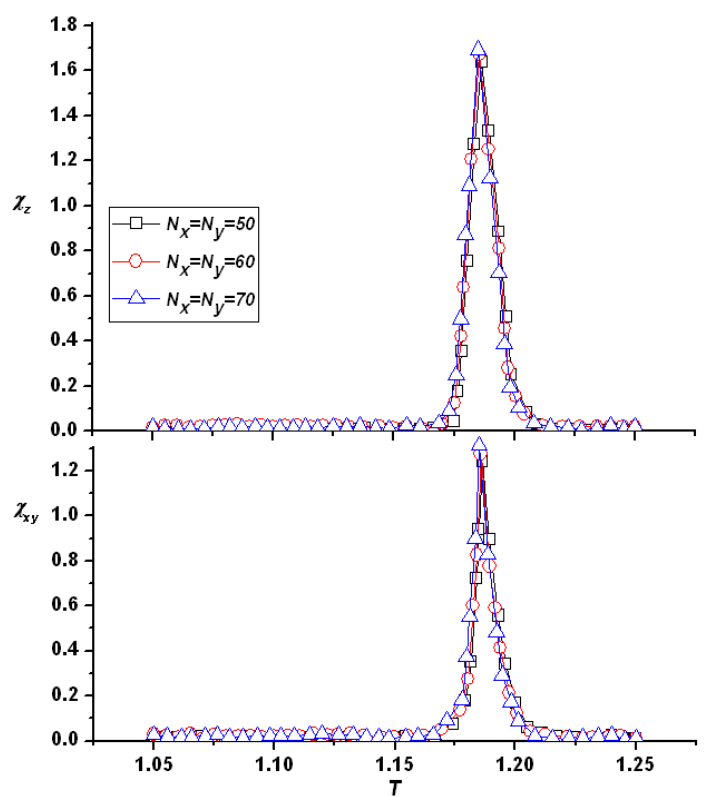

Fig. 12. Out-of-plane (top) and in-plane (bottom) magnetic susceptibility of the sample versus temperature for $J_{11}=J_{22}=J=1, J_{01}=J_{12}=J^{\prime}=0.75$ $A_{11}=A_{22}=A=1, A_{01}=A_{12}=A^{\prime}=1.3$, $D_{11}=D_{22}=D=0.175, D_{01}=D_{12}=D^{\prime}=0.2$ for different lattice size values.

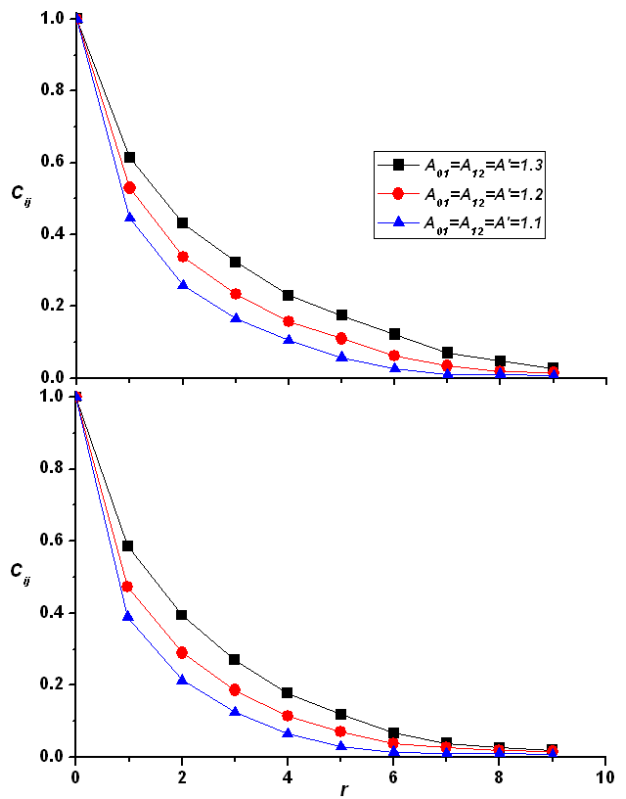

Fig. 13. Correlation function of the sample for $J_{11}=$ $J_{22}=J=1, J_{01}=J_{12}=J^{\prime}=0.75, A_{11}=A_{22}=$ $A=1, D_{11}=D_{22}=D=0.175, D_{01}=D_{12}=D^{\prime}=0.2$, for different $A_{01}=A_{12}=A^{\prime}$ values, at $T=0.85$ (top) and $T=1.15$ (bottom).

along with the lattice size enlargement) in comparison with the relative variation of the magnetic susceptibility peak's amplitude and one can therefore say that for relative large lattice size limit, the critical temperature is quite stable. Thus, one can conclude that the interface 
discontinuity of the model parameters (considered in this section) and the competition between the anisotropic and dipolar interactions induce the in-plane spin's reorientation along with increasing temperature, which practically is equivalent with a temperature dependent out-of-plane magnetic anisotropy occurrence in the system [19].

We also investigate the correlation function corresponding to the mixed phase for two different temperatures, in order to estimate the dimension of the ferromagnetic domains (Fig. 13). One observes a more abrupt decrement of the spin-spin correlation function among the classical spins given by the expression $(r$ being the coordination number):

$$
C_{r}=\sum_{i} \boldsymbol{S}_{i} \boldsymbol{S}_{i+r}
$$

as the temperature increases from $T=0.85$ to $T=1.15$, being evident that the interlayer anisotropy interaction favors the ferromagnetic arrangement of the lattice spins (the correlation function increases along with $A^{\prime}$ increasing).

\subsection{Comparative approach}

Finally, in order to emphasize the different influence of the antiferromagnetic basal layer on the superior layers of this kind of lattices, we make a comparative approach regarding the magnetic behavior of four various systems: the superior layer of the double layer lattice (model used in our previous paper [18]), the two superior layers of the triple layer lattice (threaded as a whole system) and the superior layers (1 and 2) of the triple layer lattice (treated separately), in the ferromagnetic region of the phase diagram, in order to find out the antiferromagnetic basal layer influence on these four particular systems. Thus, in Fig. 14 we show the magnetization (top) and the out-of-plane magnetic susceptibility (bottom) for the above mentioned physical systems vs. temperature for the same parameters values.

Taking into account the critical temperature associated with the transition ferromagnetism-disordered phase, one can observe that the stability of the ferromagnetic phase is different for the four systems. This is due to the different influence of the basal layer on the four studied magnetic systems. The least influence of the basal layer is observable in the case of the layer 2 (of the triple layer lattice) in comparison with the other three systems we have in view. Thus, we remark the asymmetric magnetic behavior of the two layers of the triple layer film (the basal layer influence on the layer 1 is more acute than on the layer 2). Using the presented results, we can appreciate the importance of the lattice structure respecting the ferromagnetic ordering of the investigated magnetic systems. It is evident that the antiferromagnetic basal layer influence decreases with the increase in the number of layers. Thus, for the same physical parameter values the critical temperature corresponding to FM-PM transition is shifted to lower temperature for the double layer film in comparison with the triple layer system.

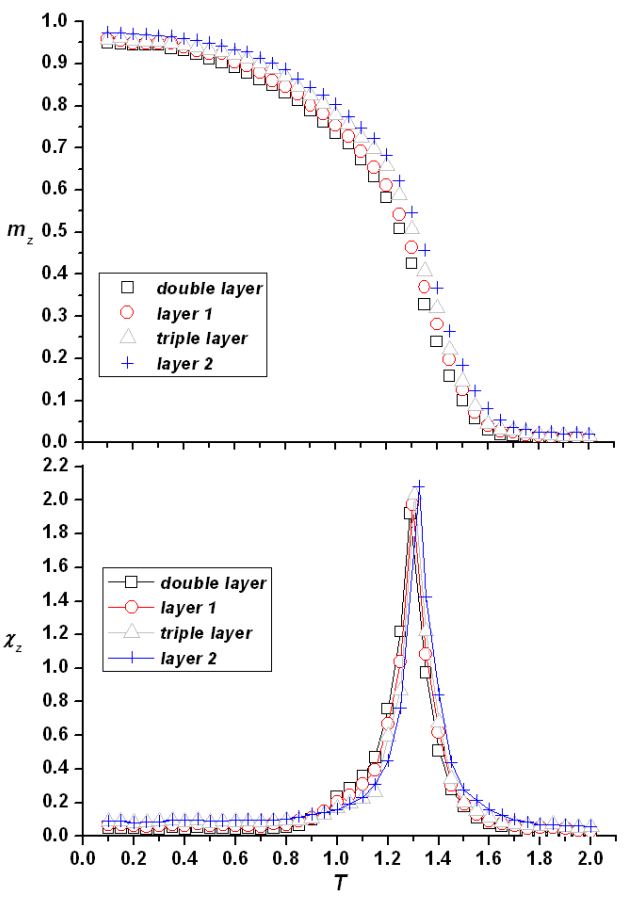

Fig. 14. Out-of-plane magnetization (top) and magnetic susceptibility (bottom) of the four mentioned systems versus temperature for $J_{11}=J_{22}=J=1$, $J_{01}=J_{12}=J^{\prime}=0.75, A_{11}=A_{22}=A=1$, $A_{01}=A_{12}=A^{\prime}=1.2, D_{11}=D_{22}=D=0.1$, $D_{01}=D_{12}=D^{\prime}=0.1$.

\section{Conclusions}

The increasing interest in nanostructured magnetic materials has been driven by demands for their practical applications as well as by their scientific importance in the last decades. Remarkable progress has been made on their technological applications, particularly for magnetic and spintronic devices such as high-density data storage and magnetic field sensors. In this paper we concern triple layer thin film magnetic properties whose basal layer is characterized by a fixed antiferromagnetic spins ordering, using Monte Carlo simulations. The basal layer's antiferromagnetic ordering influence on the sample (the other two layers) was investigated and one relieves the magnetic phases ordering occurrence for different physical model's parameters. We have focused our attention on three magnetic phase transitions: $\mathrm{FM} \rightarrow \mathrm{PM}$, $\mathrm{AFM} \rightarrow \mathrm{MP}, \mathrm{MP} \rightarrow X Y$, by calculating the interesting physical quantities of the system: the out-of-plane and in-plane magnetization, the out-of-plane and in-plane magnetic susceptibility, the specific heat and the out-of-plane staggered magnetization. In each case we have made a comparative treatment respecting the relative variation of critical temperature and of magnetic susceptibility peak. The simulations results evidence the antiferromagnetic ordering phase and the mixed phase domains appearance in the phase diagram, as a consequence of the basal layer spins antiferromagnetic ordering influ- 
ence. We have also investigated the correlation function corresponding to the mixed phase for two different temperatures, in order to estimate the dimension of the ferromagnetic domains in this case and we observed a more abrupt decrement of the spin-spin correlation function among the classical spins as the temperature increases and the correlation function enlargement along with $A^{\prime}$ increment. Finally, we have made a comparative study regarding the magnetic ordering, in order to find out the antiferromagnetic basal layer different influence on four particular magnetic systems, in the ferromagnetic region of the phase diagram.

\section{Acknowledgments}

I am very grateful to Zoltán Néda (Babes-Bolyai University of Cluj-Napoca) for extremely enlightening discussions on these subjects.

\section{References}

[1] P. Grünberg, R. Schreiber, Y. Pang, M.B. Brodsky, H. Sowers, Phys. Rev. Lett. 57, 2442 (1986).

[2] F. Saurenbach, U. Walz, L. Hinchey, P. Grünberg, W. Zinn, J. Appl. Phys. 63, 3473 (1988).

[3] R. Allenspach, J. Magn. Magn. Mater. 129, 160 (1994).

[4] M. Lévy, Solid State Phys. 47, 367 (1994).

[5] L. Néel, J. Phys. Radium 15, 376 (1954).
[6] J.G. Richter, Phys. Rev. Lett. 56, 2728 (1986).

[7] A.B. Johnston, J.N. Chapman, B. Khamsehpour, C.D.W. Wilkinson, J. Phys. D 29, 1419 (1996).

[8] M. Hehn, J.-P. Boucher, F. Roousseaux, D. Decamini, B. Bartenlian, C. Chappert, Science 272, 1789 (1996)

[9] S.T. Chui, V.N. Ryzhov, Phys. Rev. Lett. 78, 2224 (1997).

[10] E.Yu. Vedmedenko, A. Ghazali, J.-C.S. Lévy, Surf. Sci. 402-404, 391 (1998).

[11] P.J. van der Zaag, A.R. Ball, L.F. Feiner, R.M. Wolf, P.A.A. van der Heijden, J. Appl. Phys. 79, 5103 (1996).

[12] J. van Driel, F.R. de Boer, K.-M.H. Lenssen, R. Coehoorn, J. Appl. Phys. 88, 975 (2000).

[13] P.J. van der Zaag, Y. Ijiri, J.A. Borchers, L.F. Feiner, R.M. Wolf, J.M. Gaines, R.W. Erwin, M.A. Verheijen, Phys. Rev. Lett. 84, 6102 (2000).

[14] D.P. Landau, K. Binder, A Guide to Monte Carlo Simulations in Statistical Physics, Cambridge University Press, Cambridge, England 2002.

[15] Monte Carlo Methods in Statistical Physics, Ed. K. Binder, Springer-Verlag, Berlin 1979.

[16] N. Metropolis, A.W. Rosenbluth, M.N. Rosenbluth, A.H. Teller, E. Teller, J. Chem. Phys. 21, 1087 (1953).

[17] S.H. Tsai, D.P. Landau, T.C. Schulthess, J. Appl. Phys. 93, 8612 (2003).

[18] E. Bîrsan, R. Chis, A. Dobrita, Acta Phys. Pol. A 113, 1701 (2008).

[19] P.J. Jensen, H. Dreysse, Phys. Rev. B 66, 220407 (2002). 